\title{
HI in the outskirts of Nearby Spirals
}

\author{
Elias Brinks ${ }^{1}$ and António Portas ${ }^{2,1}$ \\ ${ }^{1}$ Centre for Astrophysics Research, \\ University of Hertfordshire, Hatfield AL10 9AB, United Kingdom \\ email: e.brinks@herts.ac.uk \\ ${ }^{2}$ Marketing Department, Leeds Beckett University, \\ Leeds LS1 3HE, United Kingdom \\ email: A.Portas@leedsbeckett.ac.uk
}

\begin{abstract}
We analyse nine galaxies taken from the THINGS survey to investigate the H I extent of spiral galaxy disks. We exploit the high spatial and velocity resolution, and the sensitivity of THINGS to investigate where the atomic gas disks end and what might shape their outskirts. We find that the atomic gas surface density across most of the disk is constant at 5 to $10 \mathrm{M}_{\odot} \mathrm{pc}^{-2}$ and declines at large radius. The shape of the $\mathrm{H} \mathrm{I}$ distribution can be described by a Sérsic-type function with a slope index $n=0.18-0.36$. The H I column density at which radial profiles turn over is found to be at too high a level for it to be caused by ionisation by a meta-galactic UV field. Instead we suggest the H I extent is rather set by how galaxy disks form.
\end{abstract}

Keywords. galaxies: structure, galaxies: ISM

\section{Introduction}

Spiral galaxies show a finite extent in deep H I images which means that either i) the column density drops below the detection limit; ii) the galaxies are running out of material (so the H I extent is a consequence of the process of galaxy formation); iii) there is a tidal radius beyond which material can be easily removed through interactions; iv) HI turns very cold and becomes $\mathrm{H}_{2}$ (Combes \& Pfenniger 1997); or v) the H I becomes ionised, for example by an isotropic meta-galactic UV field (Maloney 1993). These conditions are not mutually exclusive and a combination of some of the above might actually define the shape of the H I distribution at the galaxy edge.

Deep observations of neutral hydrogen down to surface brightnesses as low as $10^{19} \mathrm{~cm}^{-2}$ have shown galaxies to have a well defined extent (excluding those which are obviously interacting) and not grow indefinitely with deeper observations. For example, Irwin et al. (2009) published a map of NGC 2903 obtained with the ALFA receiver at Arecibo reaching as deep as $10^{18} \mathrm{~cm}^{-2}$, showing a ratio of $\mathrm{HI}$ diameter versus optical of 3.2. The large Arecibo beam of almost $4^{\prime}$ limits the linear resolution to $10 \mathrm{kpc}$ which is too coarse to study the detailed shape of the outskirts of this object, though.

More recently, the following projects spatially resolve the ISM in galaxy outskirts to varying degree: HALOGAS (Heald et al. 2011), Bluedisk (Wang et al. 2013), and LITTLE THINGS (Hunter et al. 2012). In parallel, cosmological simulations, e.g., EAGLE (Schaye et al. 2015) modelling galaxy formation and evolution down to the current time have reached resolutions comparable to state of the art observations (Bahé et al. 2016).

In this paper we investigate in a systematic way the shape of the outer reaches of spiral galaxies, tracing their neutral hydrogen distribution using high resolution maps from THINGS, The HI Nearby Galaxies Survey (Walter et al. 2008). This survey provides high spatial $\left(6^{\prime \prime}\right)$ and velocity $\left(5 \mathrm{~km} \mathrm{~s}^{-1}\right)$ resolution data on 34 nearby galaxies obtained with the VLA. We selected those galaxies which fit comfortably in the VLA primary 


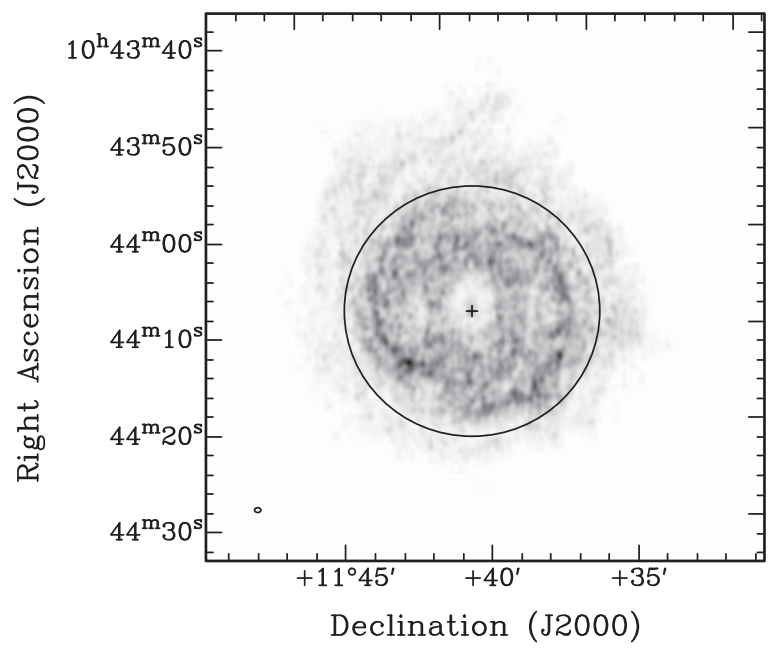

Figure 1. Deprojected H I integrated map of NGC 3351. The major axis is oriented East-West. The circle represents a radius of $15 \mathrm{kpc}$; the plus sign indicates the kinematical centre. The size of the beam is shown in the lower left-hand corner.

beam (32' in diameter) to ensure their outskirts are fully mapped. This reduced the sample to 9 objects: NGC 925, NGC 3184, NGC 3198, NGC 3351, NGC 3521, NGC 3627, NGC 5194, NGC 7331, and NGC 7773. The original data cubes were convolved to a $10^{\prime \prime}$ circular beam to avoid any bias due to beam shape and improve the signal-to-noise for faint, extended emission. At the distance of our targets, $10^{\prime \prime}$ corresponds to a linear resolution ranging from 190 to $710 \mathrm{pc}$.

\section{Results}

Fig. 1 shows the integrated HI map of NGC 3351 (M95), deprojected to a face-on orientation. The deprojected image emphasises the irregular and lopsided nature of the neutral atomic gas in the galaxy outskirts. The THINGS data reach down to surface densities of $2.5 \times 10^{19} \mathrm{~cm}^{-2}$. Naively, to increase the sensitivity one might suggest to simply produce a radial profile integrating in annuli in the plane of the galaxy. In practice, this does not work, because if we were to average the H I surface density map in rings over $360^{\circ}$ in azimuth we would in the outskirts lump together areas with and without emission, leading at best to a washing out of the signature of any edge, but more likely to a derived H I column density profile which is not a reflection of its intrinsic shape. Instead of averaging along the entire circumference, we chose a $30^{\circ}$ sector centred on the major axis and averaged along a $30^{\circ}$ wide arc in $10^{\prime \prime}$ radial bins. The decrease in signal-to-noise ratio when moving outwards is compensated for by the effect of averaging over larger areas at the periphery of the galaxy.

Fig. 2 shows in the left-hand panel the behaviour of column density with radius in a log-linear plot. We fit a modified Sérsic-type power law as follows:

$$
I(r)=I(0) e^{-(1-n)\left(\frac{r}{r_{i}}\right)^{1 / n}}
$$

with $r$ the radius and $n$ the Sérsic index. For values of $n<1, r_{i}$ is defined as the radius where the slope reaches maximum steepness before easing off. This occurs where $\partial^{2} I(r) / \partial r^{2}=0$. The line in Fig. 2 shows the Sérsic-type fit where we only used radii corresponding to the grey dots (with error bar) as they correspond to those radii where 

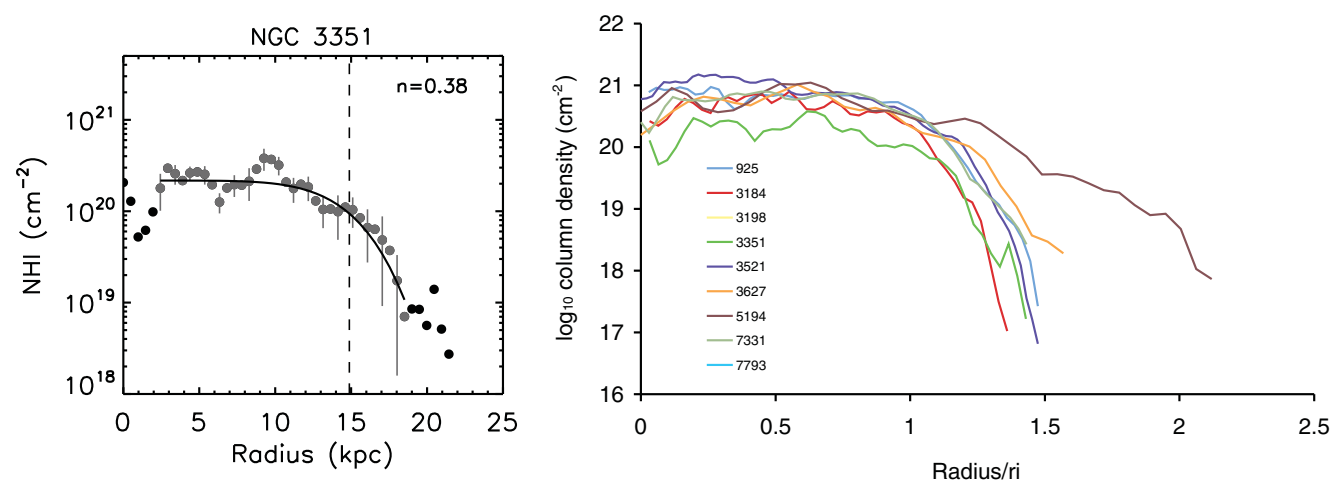

Figure 2. Left: H I column density as a function of radius in a $30^{\circ}$ sector around the receding major axis on a logarithmic intensity scale. The drawn curve is the Sérsic-type fit to the grey points only (see text for details). The characteristic radius, $r_{i}$, is represented by the vertical dashed line. Right: Radial profiles derived in a $30^{\circ}$ sector around the major axis of the 9 galaxies in our subsample. The profiles have been scaled to their respective characteristic radius, $r_{i}$. The outlier is M 51 where the $\mathrm{HI}$ is disturbed because of the interaction with its dwarf companion.

we had enough independent data points of a sufficient signal-to-noise. The black dots are more uncertain and were not included in the Sérsic-type fit. Sérsic-type fits to the profiles of all targets lead to values for $n$ ranging from $0.18-0.36$.

In the right-hand panel of Fig. 2 we show the radial profiles obtained as described above, for all 9 galaxies of our subsample, but normalised to their characteristic radius $r_{i}$. The H I profiles are remarkably similar. All profiles are flat for $r<r_{i}$, at a limiting column density corresponding to $\sim 10 \mathrm{M}_{\odot} \mathrm{pc}^{-2}$ (Bigiel et al. 2008). They all have the same characteristic turn-over to a steep decline. We find that at a column density of $5 \times 10^{19} \mathrm{~cm}^{-2}$ the H I disk has reached $85 \%$ of its maximum extent. We also see that the $\mathrm{H}$ I column density at which the radial profiles turn over is found to be at too high a level for it to be caused by ionisation by a meta-galactic UV field and we postulate that it is instead set by how galaxy disks form. This is corroborated by recent simulations of the evolution of the gas content and distribution in cosmological simulations that implement much improved sub-grid physics prescriptions (e.g., Bahé et al. 2016). The resulting simulated radial profiles are remarkably similar to those observed, i.e., confirming that their overall shape is determined by galaxy evolution, ionisation by the meta-galactic radiation only playing a rôle at column densities below a few $\times 10^{19} \mathrm{~cm}^{-2}$.

\section{References}

Bahé, Y. et al. 2016, MNRAS, 456, 1115

Bigiel, F. et al. 2008, AJ, 136, 2846

Combes, F. \& Pfenniger, D. 1997, A\& $A, 327,453$

Heald, G. et al. 2011, A\& $A, 526$, A118

Hunter, D. A. et al. 2012, AJ, 144, 143

Irwin, J. A., et al. 2009, ApJ, 692, 1447

Maloney, P. 1993, ApJ, 342, 1093

Schaye, J. et al. 2015, MNRAS, 446, 521

Walter, F. et al. $2008, A J, 136, .2563$

Wang, J. et al. 2013, MNRAS, 433, 270 